\title{
Development of Reconstruction Method for Highly Precise Shave-Off Depth Profiling*
}

\author{
Makiko Fujii ${ }^{\dagger}$ \\ Institute of Industrial Science, The University of Tokyo, \\ 4-6-1 Komaba, Meguro-ku, Tokyo 153-8505, Japan \\ Masashi Nojima \\ Faculty of Science and Technology, Tokyo University of Science, \\ 2641 Yamazaki, Noda, Chiba 278-8510, Japan and \\ Research Institute of Science and Technology, Tokyo University of Science, 2641 Yamazaki, Noda, Chiba 278-8510, Japan \\ Masanori Owari \\ Institute of Industrial Science, The University of Tokyo, \\ 4-6-1 Komaba, Meguro-ku, Tokyo 153-8505, Japan and \\ Environmental Science Center, The University of Tokyo, \\ 7-3-1 Hongo, Bunkyo-ku, Tokyo 113-0033, Japan \\ Yoshimasa Nihei \\ Faculty of Science and Technology, Tokyo University of Science, 2641 Yamazaki, Noda, Chiba 278-8510, Japan \\ (Received 10 January 2010; Accepted 28 February 2010; Published 15 May 2010)
}

\begin{abstract}
Shave-off depth profiling utilizes a focused ion beam (FIB) micro-machining process to provide the depth profile. This method is a very unique depth profiling for acquiring a depth profile by the shave-off scanning mode (The fast horizontal sweep of FIB is combined with the very slow vertical sweep). Shave-off depth profiling has its own features, absolute depth scale, pin point depth profiling and application for rough surface and hetero interface. However, the shave-off depth profile is affected by the long tail of FIB because shave-off scanning mode has the distinctive position of the primary ion beam against the sample. In this study, we acquired the different shave-off depth profiles by changing the speed of shave-off scan. Then, we estimated the approximate intensity profile of FIB by the change of the depth resolution of experimental depth profiles. By using this estimated intensity profile of FIB, we carried out the deconvolution to remove the influence of the long tails of FIB. As a result, this new data analysis method enables us to reconstruct highly precise calculated depth profile that reflects not the intensity profile of FIB but the true elemental distribution. [DOI: 10.1380/ejssnt.2010.237]
\end{abstract}

Keywords: Secondary ion mass spectrometry; Shave-off depth profiling; Focused ion beam

\section{INTRODUCTION}

In recent years, the establishment of local part analytical methods in a limited area is aspired for a semiconductor and an electronic device industry. We have been developing shave-off depth profiling with the nano-beam secondary ion mass spectrometer (nano-beam SIMS), one of the powerful methods that can be applied for analyzing such materials and devices. Shave-off depth profiling has its own features, absolute scale, pin point depth profiling and application for rough surface and hetero interface. However, shave-off depth profiling has the following problem to be solved. The leading edge and trailing edge of the acquired shave-off depth profile have particular shapes because the shave-off profile is strongly affected by long tails outside of focused ion beam (FIB). The depth resolution of the shave-off depth profile was about $40 \mathrm{~nm}$ in our case [1]. When the thickness of the film is sufficient, it is easy to obtain the depth distribution in the film directly from the acquired profile. In contrast, when the thickness is not sufficient, it is difficult to obtain the concentration gradient directly from the profile because the profile in-

*This paper was presented at 7th International Symposium on Atomic Level Characterizations for New Materials and Devices, The Westin Maui Resort \& Spa, Hawaii, U.S.A., 6-11 December, 2009.

†Corresponding author: m-fujii@iis.u-tokyo.ac.jp cludes not only the effect of the concentration gradient, but also the effect of the long tails of the FIB. Toi et al. has reported about the reduction of the influence of the long tails by using the protection film [1]. In our previous study, we proposed the shave-off scanning speed as one of the factors that affect the long tails of FIB [2]. In this study, we newly propose the reconstruction method that enables us to remove the influence of the long tail of FIB from acquired shave-off depth profile and accomplished highly precise shave-off depth profiling.

\section{EXPERIMENTAL}

\section{A. Shave-off depth profiling}

Different shave-off depth profiles were acquired by changing the experimental condition on speed of the shave-off scan. In this study, the samples were the pieces of a multi-layer thin film ( $\mathrm{Al} 1 \mu \mathrm{m} / \mathrm{SiO}_{2} 0.8 \mu \mathrm{m} / \mathrm{Si}$ substrate) prepared by FIB micro sampling technique [3]. The size of the thinned samples was $6.5 \mu \mathrm{m}$ in width, $1 \mu \mathrm{m}$ in thickness and $6 \mu \mathrm{m}$ in height. A carbon film of about $0.3 \mu \mathrm{m}$ thickness was deposited on the sidewall of the sample in order to protect the sample from damage by the long tail of FIB during shave-off depth profiling [4]. This multi-layer thin film is the first product in the semiconductor manufacturing process. Figure 1 shows a SEM image of the piece of multi-layer thin film picked-up by 


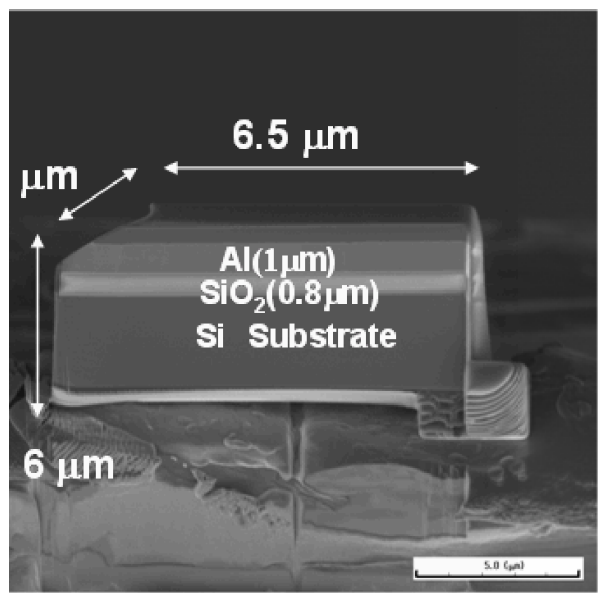

FIG. 1: SEM image of sample picked-up from multi-layer thin film by FIB.

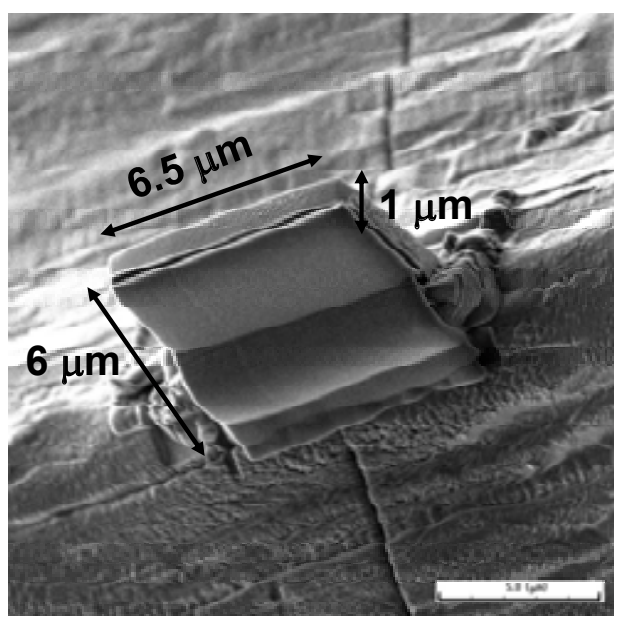

FIG. 2: SEM image of sample after depositing the carbon film.

FIB before depositing carbon film. Figure 2 shows the same sample after depositing the carbon film.

The primary ions of this apparatus were field-emitted $\mathrm{Ga}^{+}$with $30 \mathrm{keV}$ acceleration energy and $35 \mathrm{pA}$ beam current. The mass analyzer was a modified Mattaugh-Herzog type that is originally equipped with a spark source mass spectrograph (JEOL JMS01BM). This apparatus realizes nano-dimensional elemental mapping and shave-off depth profiling. Shave-off depth profiling is a very unique depth profiling method for acquiring a depth profile by the shave-off scanning mode (The fast horizontal sweep of FIB is combined with the very slow vertical sweep). Figure 3 is the schematic of shave-off depth profiling process.

\section{B. Simulation}

We assumed that the shave-off depth profile is essentially a convolution of the beam profile with the true elemental distribution [4]. Thus, the signal intensity $I(x)$

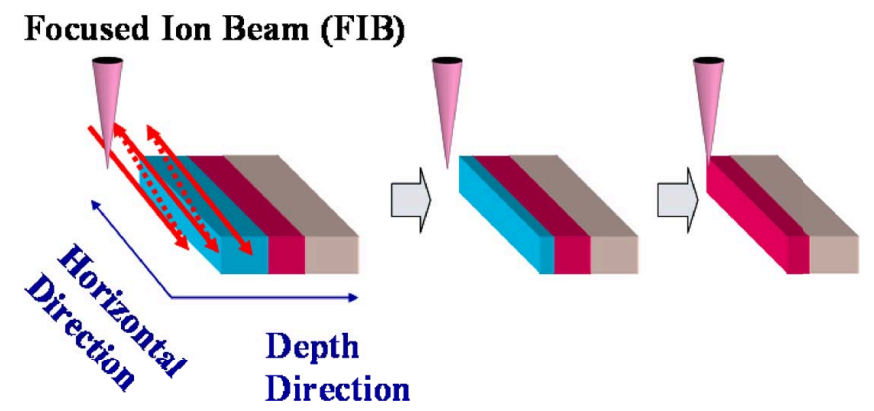

FIG. 3: The schematic of shave-off depth profiling process.

can be calculated as:

$$
I(x)=I_{0} \int_{x+\alpha}^{x+\beta} N_{0}(x) G(x-\mu) d x .
$$

Here, $\mu$ is the center position of FIB moving into the direction of depth at a constant speed. $x$ is the depth position with its origin at an arbitrary point and $N_{0}(x)$ is the concentration of the target atom at depth $x . G(x-\mu)$ is the intensity profile of the primary ion beam. $\alpha$ is a parameter indicating a position where the sample is shaved off completely. This is because the sample is shaved off by not all the intensity of the beam but the only few part of the beam near the long tail. When the beam is approaching the sample, the upper side of the sample is shaved off little by little. When the beam approached the sample sufficiently, all the parts of sample are shaved off by a constant intensity of the beam. $\beta$ is the parameter related to the thickness of the carbon film.

We also proposed the method of simulating the depth resolution by changing the speed of shave-off scan [2]. The same amount of exposure of the primary ion beam is needed for sputtering the sample of the same volume. Therefore, if the scanning speed is doubled, the amount of the primary ion beam exposing to the sample in the unit time has to be doubled. The value of $\alpha$ changes along with it because the position of $\alpha$ also expresses the amount of the primary ion beam exposing the sample in the unit time. Figure 4 shows the position of $\alpha$ with the scanning speed. The black area of Fig. 3 expresses the amount of the primary ion beam exposing the sample in the unit time. We presumed the certain scanning speed "normal speed" and discussed about the change of the scanning speed. Then, the scanning speed becomes twice faster, the black area of Fig. 4 becomes twice larger. Thus, the faster the scanning speed of FIB the closer $\alpha$ moves toward negative direction.

In this assumption, the degree of the change of $\alpha$ only depends on the scanning speed and the intensity profile of FIB. Thus, we can estimate the approximate intensity profile of FIB by the change of $\alpha$ and deconvolute the observed profile based on above equation to remove the influence of the long tail of FIB in order to acquire the true elemental distribution. 

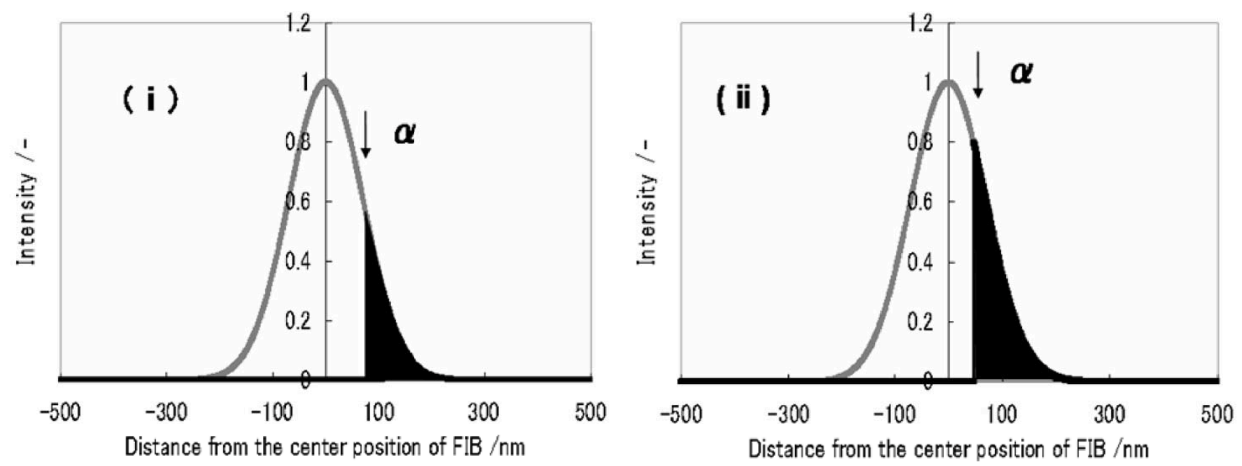

FIG. 4: The position of $\alpha$ with the scanning speed; (i) "normal speed", (ii) twice faster.

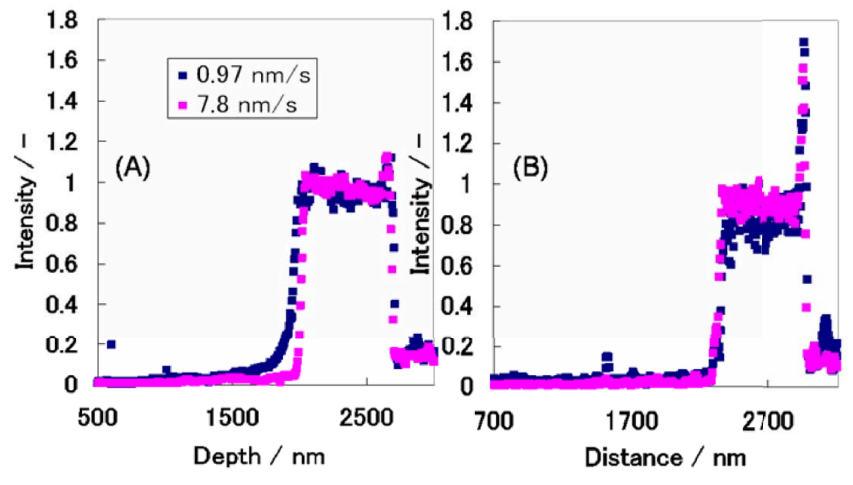

FIG. 5: (A) The acquired shave-off depth profiles. (B) The depth profiles after the deconvolution.

\section{RESULTS AND DISCUSSIONS}

We acquired some different shave-of depth profiles by changing the experimental condition on the speed of shave-off scan. Figure 5(A) shows the acquired shaveoff depth profiles of $\mathrm{Si}^{+}$on the scanning speed $0.97 \mathrm{~nm} / \mathrm{s}$ and $7.8 \mathrm{~nm} / \mathrm{s}$, respectively. We defined the depth resolution as the distance between depths with $16 \%$ and $84 \%$ of the maximum secondary ion intensity. According to this criterion, depth resolutions at these two depth profiles were estimated to be about $120 \mathrm{~nm}$ and $50 \mathrm{~nm}$ for the scanning speed $0.97 \mathrm{~nm} / \mathrm{s}$ and $7.8 \mathrm{~nm} / \mathrm{s}$, respectively. This clearly indicates that the faster the speed of shaveoff scan becomes, the smaller the influence of a long tail of FIB changes.

Then, we estimated the approximate intensity profile of FIB by the change of depth resolution. In previous study, we reported that the intensity profile of FIB is more similar to the convolution of Gaussian with Lorenzian than Gaussian [2]. We carried out the method of least squares fitting with the parameter of $\alpha$ and FWHM of Gaussian and Lorenzian to determine the intensity profile of the beam which is assumed to be the distribution indicates the most similar change of depth resolution to the experimental one. The estimated intensity profile of FIB at this experimental condition was the convolution of Gaussian
30 nm FWHM with Lorenzian 15 nm FWHM.

By using above estimated intensity profile of FIB, we carried out deconvolution of the above equation to remove the influence of the long tail of FIB and acquired the true elemental distribution. Figure 5(B) shows the depth profiles after the deconvolution. The deconvolution process shifts the depth profiles depending on the assumed beam profile. Therefore, absolute depth scale has no special meanings. The depth resolutions at these depth profiles of (B) were both estimated to be $20 \mathrm{~nm}$ by using the least squares fitting. These two depth profiles have very similar shape of the leading edge. These results suggest that the effect of the long tail of FIB was removed almost completely. Moreover, there are sharp peaks at the $\mathrm{SiO}_{2} / \mathrm{Si}$ interface of both profiles after deconvolution. Though the information we can acquire from these profiles is not sufficient but they can be originated to be migrated $\mathrm{Si}$ from $\mathrm{Si}$ part to $\mathrm{SiO}_{2}$ part because $\mathrm{Si}$ has larger atom density than $\mathrm{SiO}_{2}$. These sharp peaks could not be distinguished by experimental profiles because of insufficiency of the depth resolution. This new reconstruction method enabled us to acquire more detailed depth distribution of the sample.

\section{CONCLUSIONS}

We acquired different shave-off depth profiles by changing the speed of shave-off scan. Then, we estimated the approximate intensity profile of FIB by the change of the depth resolution of the experimental depth profiles. By using this estimated intensity profile of FIB, we carried out the deconvolution. As a result, this new data analysis method enabled us to reconstruct highly precise calculated depth profile that reflects true elemental distribution from the experimental shave-off depth profile.

\section{Acknowledgments}

We would thank to Ms. Tomoko Arimitsu and Ms. Ikuko Nakatani of SII Nanotechnology and Mr. Kazuto Nakamura of TOSOH Analysis and Research Center for technical support and helpful suggestions. 
[1] M. Toi, A. Maekawa, T. Yamamoto, B. Tomiyasu, T. Sakamoto, M. Owari, M. Nojima, and Y. Nihei, J. Surf. Anal. 12, 170 (2005).

[2] M. Fujii, K. Nakamura, Y. Ishizaki, M. Nojima, M. Owari, and Y. Nihei, Appl. Surf. Sci. 255, 1354 (2008).

[3] F. A. Stevie, C. B. Vartuli, L. A. Giannuzzi, T. L. Shofner, S. R. Brown, B. Rossie, F. Hillion, R. H. Mills, M. Antonell,
R. B. Irwin, and B. M. Purcell, Surf. Interface Anal. 31, 345 (2001).

[4] K. Nakamura, Y. Ishikawa, K. Utsumi, H. Igusa, R. Tanaka, Y. Ishizaki, T. Yamamoto, A. Maekawa, M. Owari, M. Nojima, and Y. Nihei, Surf. Interface Anal. 38, 1734 (2006). 